\title{
Usability and the Semantic Web
}

\author{
Anthony Jameson \\ DFKI - German Research Center for Artificial Intelligence \\ and International University in Germany \\ http://dfki.de/ jameson/
}

\begin{abstract}
In addition to its technical implications, the semantic web vision gives rise to some challenges concerning usability and interface design. What difficulties can arise when persons with little or no relevant training try to (a) formulate knowledge (e.g., with ontology editors or annotation tools) in such a way that it can be exploited by semantic web technologies; or (b) leverage semantic information while querying or browsing? What strategies have been applied in an effort to overcome these difficulties, and what are the main open issues that remain? This talk will address these questions, referring to examples and results from a variety of research efforts, including the project SemIPort, which concerns semantic methods and tools for information portals, and Halo 2, in which tools have been developed and evaluated that enable scientists to formalize and query college-level scientific knowledge.
\end{abstract}

